\title{
Principios de la investigación en ciencias naturales históricas: ¿Por qué en biología es necesaria la Historia Natural de los organismos?
}

\section{Principles of research in historical Natural Sciences: Why is the Natural History of organisms necessary in Biology?}

\author{
Carolina Villagrán ${ }^{1 *}$, Ricardo Segovia ${ }^{2,3}$ \& Lucia Castillo² \\ ${ }^{1}$ Departamento de Biología, Facultad de Ciencias, Universidad de Chile, Las Pameras \#3425, Ñuñoa, Santiago de Chile. \\ ${ }^{2}$ Departamento de Ecología, Facultad de Ciencias, Universidad de Chile, Las Pameras \#3425, Ñuñoa, Santiago de Chile. \\ ${ }^{3}$ Instituto de Ecología y Biodiversidad, IEB, Las Pameras \#3425, Ñuñoa, Santiago de Chile. \\ *symphyogina@hotmail.com
}

\begin{abstract}
RESUMEN
Se discute en este trabajo el desarrollo histórico, significado y valor heurístico de la Historia Natural en la reflexión teórica y generación de hipótesis en biología. Sobre esta base se fundamenta la validez de una metodología propia en el dominio de las ciencias naturales históricas, en general. Primero se examinan de manera crítica los principios generales y metodologías que rigen la investigación en ciencias naturales históricas, en contraste con las ciencias biológicas experimentales. Se comenta el actualismo, un principio fundamental que valida el uso de análogos actuales y la formulación de retrodicciones en la investigación del pasado. También se comentan brevemente los métodos más comunes de la investigación histórica, como la iteración de patrones procedentes de distintas fuentes de datos paleoambientales (proxy data), las evidencias correlativas que permiten reconstruir condiciones paleoecológicas a través de series de tiempo. Se discute la validez del principio de causa común en el juicio e interpretación histórica de acontecimientos pasados sobre la base de una causalidad temporal asimétrica (asimetría de sobredeterminación). Esta causalidad justifica la inferencia histórica (postdicción), en contraste con la predicción. En la segunda parte de este trabajo se consideran preguntas específicas sobre Historia Natural de los organismos. Con este propósito se introducen brevemente los principios de autoorganización y acoplamiento estructural organismo-espacio de vida, ideas heurísticas que fundamentan conceptos y metodologías propias en los estudios de los seres vivos (como adaptación, homología, analogía, anomalía estructural, disyunción, etc.), tanto en las investigaciones sobre desarrollo y morfología como en sistemática, filogenia, paleontología, ecología, evolución, y biogeografía. Finalmente, se destaca la importancia de la consideración de esta diversidad metodológica en ciencias naturales históricas como una contribución al debate actual acerca de la validez del campo de la Historia Natural.
\end{abstract}

Palabras clave: Actualismo, proxy data, causa común, asimetría temporal, retro-postpredicción, autoproducción, acoplamiento estructural.

\begin{abstract}
We discuss the meaning and heuristic role of Natural History in theoretical thinking and hypothesis formulation in biology. Through this considerations, we support the validation of the use of a specific methodology in the field of historical natural sciences, in general. We begin by examining critically the general methodological principles that guide research in historical natural sciences, in contrast with the experimental biological sciences. We examine actualism, a fundamental principle in the studies of the past that validates the use of contemporary analogs and retrodictions. We discuss briefly some of the methods commonly used in historical research, like pattern iteration from different series of paleoecological evidence (proxy data), the correlative evidences that enables the reconstruction of palaeoecological conditions in time series. We also discuss the principle of common cause in historical judgment and interpretation of past events, over the basis of a temporarily asymmetric causality (asymmetry of overdetermination). This causality justifies historical inferences (postdictions), in contrast with prediction. In the second part of this work, we consider the specific questions of the Natural History of organisms. We briefly introduce the concepts of self-organization and structural coupling of organismsliving space, heuristic ideas that validate the use of specific concepts and methodologies in the study of living beings (adaptation, homology, analogy, structural anomaly, disjunction, etc.), in research in development and morphology, as well as in systematics, phylogeny, paleontology, ecology, evolution, and biogeography. Finally, we highlight the importance of
\end{abstract}


methodological diversity in historical natural sciences as a contribution to the contemporary debate over the validity of the field of Natural History.

KeYwoRds: Actualism, proxy data, common cause, temporal asymmetry, retro-pre-postdiction, self-production, structural coupling

\section{INTRODUCCIÓN}

La disciplina de la "Historia Natural" nació con la búsqueda de principios que gobiernan el orden natural de los seres vivos en el siglo XVII, sobre la base de la observación metódica de los organismos mismos, esto es, sin referencia el discurso que caracterizara la época medieval, donde la observación se mezclaba con la literatura, fantasía, leyenda y utilidad. Pese a importantes diferencias entre ellos, el orden que proponen los naturalistas en el siglo XVII - como Linneo y Buffon- enfatiza la observación de la anatomía externa e interna de los organismos, método que posibilita su reconocimiento y comparación y, por ende, el desarrollo de una nomenclatura, un orden sistemático y la comunicación universal. De esta suerte, la Taxonomía se constituye en la ciencia del orden natural, donde los conceptos fundamentales son los de jerarquía, selección de caracteres y sistema. Así, lejos de constituir un campo meramente descriptivo, desorganizado y subjetivo, la Historia Natural moderna -y sus incipientes descendientes, la Botánica, Zoología, Embriología, Morfología, Anatomía, etc.- nacen inspirados en el modelo de racionalidad de las ciencias físicas y en el pensamiento mecanicista cartesiano, pensamiento que da origen a las ciencias naturales en general.

Esta mirada moderna experimenta un giro radical en el siglo XIX, cuando se reconfigura el campo de la Historia Natural sobre la base de nuevos principios y conceptos que caracterizan la transición de modernidad a la época contemporánea. Una de las ideas claves de este cambio de paradigma es la noción de "organismo", introducida por Kant en 1790. En contraste con la materia inanimada, los seres vivos son concebidos por primera vez como productos o fines naturales de un proceso de autoproducción y autoorganización. El principio de "conformidad a fin" de los seres vivos lo propone Kant como un principio reflexivo, pero necesario para orientar la investigación y la formulación de nuevas leyes empíricas (uso heurístico). Este principio surge como respuesta a la multiplicidad de formas y fenómenos contingentes del dominio de los seres vivos, contingencia que parece rebasar la posibilidad de explicación por causalidad meramente mecánica. Paralelamente, los naturalistas del siglo XIX, -como De Candolle, Jussieau, Cuvier, Lamarck, Darwin, Haeckel, Von Uexküll, entre otros- abren el espacio evolutivo-histórico de los organismos, al enfatizar en la clasificación de plantas y animales la búsqueda de aquellos caracteres naturales, capaces de revelar el plan estructural común de los grandes grupos y la función integrada del organismo y sus partes en conexión con su entorno.

En este contexto, es crucial el giro que conlleva el pensamiento evolutivo de Lamark y Darwin, quienes reemplazan la doctrina fijista de las especies, dominante en su época, por una concepción que reconoce sus transformaciones como producto de la historia. La teoría evolutiva -fundamentada en la integración y discusión de evidencias empíricas procedentes de la Historia Natural de la época (sistemática, anatomía comparada, desarrollo, distribución geográfica, geología y paleontología)- se plasma en El Origen de las Especies (1859), la obra cumbre de Darwin, que transforma radicalmente el modo de entender las relaciones entre los organismos e introduce una perspectiva histórica en el estudio de la vida. Así la Historia Natural se abre hacia la comprensión de los seres vivos como agentes de su propia historia. El tiempo irrumpe en el ordenado espacio de la clasificación de la modernidad, centrada en la búsqueda de una mathesis cualitativa para el ordenamiento de los seres vivos; ella cede paso a una nueva "Historia Natural", centrada ahora en el origen y transformación histórica de los mismos.

Hoy, la Historia Natural constituye un ámbito del quehacer biológico que se considera obsoleto. En nuestro país, desde hace más de una década, la Historia Natural ha sido deslegitimada como un área vigente en Ciencias Naturales (Jaksic 1999), fundamentalmente porque: a) se sostiene que muchas de sus proposiciones no pueden ser refutadas empíricamente, es decir, no pueden ser sometidas a una prueba de hipótesis de acuerdo al método experimental de la ciencia y b) se la considera un ámbito meramente descriptivo, obsoleto y sin retorno, es decir, se la retrotrae a la época de sus inicios en el medioevo, la época pre-moderna.

Respecto al cuestionamiento central que se hace a la Historia Natural, -como es un modo no válido de hacer ciencia, por la ausencia de prueba de hipótesis a través de la metodología hipotético-deductiva-, es preciso señalar que esta crítica se ha extendido muchas veces también al quehacer de la ecología, evolución, biogeografía y paleobiología (e.g. Peters 1980, Armesto 1985), o sea todos los dominios actualmente preocupados de la historia natural de los organismos. Una aplicación dogmática del método de prueba de hipótesis de Popper es en muchos casos imposible en biología, particularmente cuando se trata de procesos evolutivos donde la experiencia debe entenderse en un sentido más amplio, es decir como un 
proceso natural histórico. Así, hay conceptos fundamentales en biología que no son susceptibles de refutación empírica -e.g., los principios de selección natural, adaptación, deriva natural (Armesto 1985, Rozzi et al. 1998), etc. Por ejemplo, Armesto (1985) sostiene que "la observación de adaptación al ambiente en un organismo constituiría una prueba de la selección natural, pero la observación de un caso de desadaptación no es aceptada como una refutación de la teoría" (p. 107). Todos estos conceptos son necesarios para entender los fenómenos históricoevolutivos, pero no constituyen principios determinantes $y$, por ende, son irreductibles a explicaciones en conformidad a leyes universales. Se ha afirmado que su valor "reside en su poder heurístico que lleva a la generación de teorías e hipótesis verificables" (Armesto 1985) o que juegan un papel esencial "en el diseño acertado de observaciones y experimentos de campo" (Willson \& Armesto 2006, p. 279). Bajo esta perspectiva, los fenómenos complejos en ecología y evolución no son reductibles a diseños experimentales simples, ya que éstos enmascaran la pluricausalidad de los fenómenos.

Nuestro objetivo aquí es tomar posición en este debate para argumentar por qué la Historia Natural juega un papel heurístico fundamental en la reflexión sobre fenómenos propios de los organismos, tanto en lo referente a la formulación de principios $^{1}$ como respecto a la necesaria elección de los métodos de investigación histórica. En la primera parte presentamos y discutimos los principios y metódicas de investigación común en ciencias naturales históricas, en contraste con las así llamadas ciencias biológicas experimentales. En la segunda parte se tratan los principios que rigen la comprensión histórica de los seres vivos y la metodología propia que demanda su estudio.

1. Principios metodológicos de las ciencias naturales HISTÓRICAS

El primer punto que queremos tratar es que el carácter histórico de los organismos, que da nombre a la Historia Natural, como asimismo el estudio histórico en general, implican la aplicación de una metodología singular. Así lo exige la naturaleza de sus preguntas. ¿En qué modos de hacer ciencia reside la identidad de las ciencias naturales históricas y cuáles son sus metodologías propias? ¿De qué principios se deduce su validez? En referencia a esta temática, a continuación se presenta el principio de actualismo como pivote de las ciencias naturales históricas y se ilustra su uso metodológico.

\section{1. Principio de Actualismo}

Este principio fue formulado en el siglo XVIII por Hutton y

'Desde Aristóteles, la palabra "principio" (lat. princip̌um) se puede usar de muchas maneras. En este texto la usamos en sentido muy general para referirnos a ideas fundamentales que sirven de base para un razonamiento o para la reflexión en torno a un problema. reformulado y difundido luego como "uniformitarianismo"2 en los "Principios de Geología" de Lyell (1830-1833). Este es el primer principio formal que sirve de base a la investigación en ciencias naturales históricas desde el siglo XIX. El principio actualista sostiene que los procesos del presente son equivalentes a los que ocurrieron en el pasado, en otras palabras, que "el presente es clave para entender el pasado". $\mathrm{El}$ actualismo en ciencias naturales históricas valida el uso de "análogos actuales" para formular retrodicciones (Fig. 1), es decir, procesos que ocurren a pequeña escala espaciotemporal $-\mathrm{y}$ observables en el presente con los métodos usuales de investigación empírica- son extrapolables hacia el pasado. La base del actualismo es el estudio de relaciones accesibles en el presente y su inferencia hacia el pasado, de acuerdo a un razonamiento por analogía ${ }^{3}$. De acuerdo a Gould (1986), es Darwin quien introduce por primera vez este principio en Historia Natural. Lo aplica, por ejemplo, al analizar los experimentos de los criadores de animales y plantas, y a sus propias experiencias con selección artificial de palomas y orquídeas: observaciones a escala temporal limitada le permiten apreciar el grado de variación dentro de las poblaciones actuales y plantear así la pregunta central de su teoría: ¿Existe un proceso selectivo natural análogo a la selección artificial efectuada por el hombre?

\subsection{Metodologías de las ciencias naturales históricas}

1.2.1. ANÁlogos ACTUALES E ITERACIÓN DE SERIES DE EVIDENCIAS De acuerdo con el principio actualista, un primer momento en el estudio del pasado consiste en el uso de análogos modernos para calibrar la señal paleoambiental de los registros históricos, de acuerdo a métodos específicos según la clase de indicador usado (Fig. 1, proxy data ${ }^{4}$ ). La iteración de evidencias procedentes de distintas series de proxy constituye la base empírica sobre la cual es posible investigar el sentido de procesos históricos particulares. De acuerdo a Gould (1986), un segundo enfoque metodológico -también atribuido a Darwin- es la convergencia de patrones, esto es, la conciliación entre series de evidencias provenientes de distintas fuentes para verificar o refutar un mecanismo causal común. Darwin aplica este método

${ }^{2}$ Tanto la forma sustantiva como metodológica del uniformitarianismo de Lyell es juzgada irrelevante por Gould (1965), en tanto no difieren del método inductivo usado comúnmente en Geología.

${ }^{3}$ Analogía: Se refiere a la relación de semejanza entre cosas distintas o al razonamiento basado en atributos semejantes entre cosas diferentes (RAE)

${ }^{4}$ Se define como proxy data (proxy: sustituto, representante) cualquier registro paleoambiental utilizado en la inferencia de eventos pasados y cuyo valor indicador se infiere a partir de una herramienta metódica basada en el análisis de relaciones observables en la actualidad. Así, la extracción de la señal paleoecológica de un proxy data requiere de una calibración en función del análogo moderno (Dictionary of Geological Terms. R. L. Bates \& J. A. Jackson Anchor Books Edition 1984, USA). 
en el Origen de las especies, a saber: organización de las evidencias derivadas de la Historia Natural de su época en un sistema coherente en torno a la idea central de evolución, integrando información entregada por Buffon, Cuvier y Lamarck, entre otros, y considerando nuevos datos procedentes de la geología, climatología y de su propia experiencia de campo.

El mejor ejemplo del uso de análogos modernos en la investigación del pasado es el monumental despliegue de investigación durante la segunda mitad del siglo XX en indicadores paleoclimáticos (proxy data), tanto físicos como biológicos, en océanos, glaciares y continentes, a nivel global. La conciliación de las distintas series de evidencias empíricas ha permitido trazar los cambios climáticos del Pleistoceno al nivel global, interés gatillado por la formulación de la hipótesis astronómica u orbital de Milutin Milankovitch en 1923. Ésta interpreta los ciclos glaciales del Cuaternario sobre la base de la variación milenial de los parámetros orbitales de la Tierra y provee así el fundamento de las ciencias naturales históricas más recientes, la Paleo-climatología y la Paleo-oceanografía. La abundante evidencia empírica acumulada y el desarrollo de sofisticados métodos (por ejemplo, para la correlación y fechado de proxy, uso de isótopos estables e inestables en la estimación del paleoclima, etc.) demuestra el impulso creativo y el papel heurístico de las hipótesis históricas en ciencias naturales.

\subsubsection{CAUSA COMÚN E INFERENCIA DE LA MEJOR EXPLICACIÓN}

Recientemente, Cleland $(2001,2002,2011)$ ha revisado las distintas metodologías de las ciencias naturales históricas. Aunque las hipótesis históricas pueden ser abordadas mediante el contraste de hipótesis únicas, numerosos autores (e.g., Feyerabend 1975; Ariew 2007) proponen que la "inferencia de la mejor explicación" entre varias hipótesis rivales constituye la mejor metódica. En otras palabras, un enfoque de múltiples hipótesis concurrentes favorece la elección de aquella de la cual se infiere la "causa común" (common cause explanation) que mejor explica el conjunto de evidencias. La proliferación de hipótesis rivales favorecería un vuelco en la mirada del investigador hacia la formulación de hipótesis incompatibles con la teoría, y una reinterpretación del cuerpo de evidencias disponibles. Se invierte así el camino del actualismo y el pasado se constituye en la clave para interpretar el presente (postdicciones, Fig. 1).

La concurrencia de distintas hipótesis en la búsqueda de la causa común que mejor explica un acontecimiento ha sido fructífera en la selección de las principales hipótesis históricas contemporáneas, por ejemplo, la idea de deriva continental -formulada por Alfred Wegener en 1912- es una hipótesis que cambia la perspectiva dominante de una Tierra estática por una Tierra dinámica en que los continentes han cambiado históricamente su posición y configuración. El cúmulo de evidencias procedentes de la geología, acumuladas después de la formulación de la hipótesis de deriva continental, permitieron su replanteamiento en la década de los 60 por Hess y Dietz, como la teoría de Tectónica de Placas. Para Wegener -enfrentado a la concepción dominante- la hipótesis de una Tierra dinámica era la que mejor explicaba una serie de evidencias procedentes de diversas disciplinas (geografía, biología, geofísica, glaciología, paleontología, etc.) en torno a una idea integradora, de manera análoga al pensamiento de Darwin en biología. Como veremos, esta hipótesis tiene profundas consecuencias para la comprensión de los cambios históricos en las distribuciones de las biotas determinados por la geología.

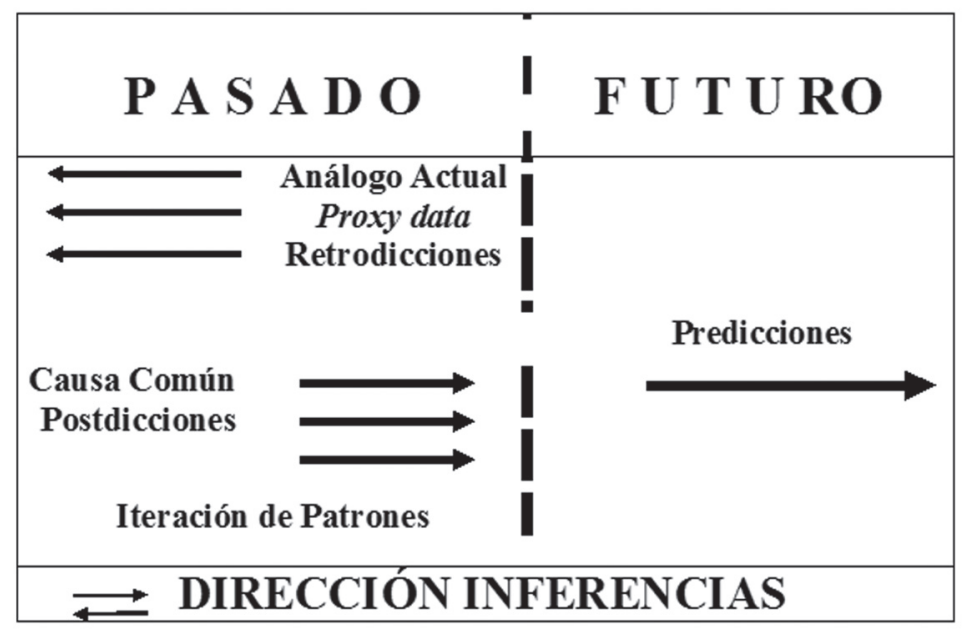

FIGURA 1: Esquema de la metodología en ciencias naturales históricas. Asimetría temporal de causación entre el pasado y el futuro.

FIgURE 1. Diagram of the methodology in historical Natural Sciences. Temporal asymmetry of causation between past and future. 
Por otra parte, Cleland (2011) ejemplifica el debate entre hipótesis explicativas rivales con referencia a la extinción masiva de finales del Cretácico y el proyecto de investigación de Álvarez et al. (1980), que permitió la inferencia de una causa común de este dramático acontecimiento y el hallazgo de la evidencia crucial: la presencia de una capa de iridio en los estratos del límite Cretácico-Terciario en varias regiones del mundo, que se atribuye al impacto de un bólido de origen extraterrestre.

\subsection{Discusión y CONCLUSiOnes Respecto A LOS ASPeCtos} METODOLÓGICOS

Sober (2001) y Turner (2004) critican el ejercicio de búsqueda de una "causa común e inferencia de la mejor explicación" con referencia a series de eventos causalmente independientes (distintas fuentes de proxy) -pero de comportamiento análogo- y califican este enfoque de metafísico. Cleland (2011) reconoce que el principio de causa común no tiene validez lógica, si no que es más bien un principio reflexivo que infiere la mejor explicación de la sobreabundancia de evidencia empírica. En otras palabras, como se trata de inferencias de causas pasadas, de procesos temporales a los cuales no tenemos acceso, se infiere su validez a través de series de efectos cuya base empírica es incuestionable. En este contexto, Cleland afirma la importancia central de la explicación por sobre la predicción en ciencias naturales históricas.

De acuerdo a Cleland (2001), la estructura de las explicaciones en ciencias naturales históricas, basada en la inferencia de la mejor explicación, difiere fundamentalmente de la lógica de la predicción. Esto porque existe una singular asimetría temporal en la naturaleza de los eventos en este dominio, en comparación con la biología experimental, lo que genera una diferencia metodológica fundamental. Se trata de la asimetría causal entre los eventos del presente, pasado y futuro (Fig. 1), en otras palabras, una causalidad temporal asimétrica que la autora denomina "asimetría de excesiva determinación" (asymmetry of Overdetermination). La tesis central es: "events in our universe are causally connected in time in an asymmetrical manner" (Cleland 2011:20). De esta manera, eventos discretos y locales determinan excesivamente (overdetermine) su causa en el pasado, ya que cada causa da origen a múltiples series de efectos, cada una de las cuales es suficiente para establecer la conexión causal con su origen. A su vez, las causas subdeterminan (subdetermine) sus efectos, ya que rara vez constituyen el único factor responsable de un efecto en particular en

${ }^{5}$ Se dice que un evento está "excesivamente determinado" (la palabra overdetermination, no tiene traducción en español) cuando el efecto observado puede ser atribuido a causas eficientes distintas y múltiples. La sobre-determinación constituye un problema para la lógica tradicional, ya que ella exige necesariamente que cualquier efecto observado sea determinado por una sola causa suficiente para validar la conclusión. el futuro. Este argumento es coherente con lo planteado por Fuentes y Sánchez (1982), quienes destacan que las postdicciones, a diferencia de las predicciones, basadas en experimentos de laboratorio que analizan una respuesta futura, "se basan en 'experimentos naturales', fenómenos que ya ocurrieron en el momento de plantear la hipótesis" (Fig. 1).

En este texto hemos querido insistir en la necesidad de reconocer una diferencia fundamental entre la metodología de las ciencias naturales históricas y la de la biología experimental, para así enfatizar la importancia fundamental que tiene el mantener en mente la consideración de un pluralismo metodológico en la investigación en el campo de la Historia Natural. En este sentido, hemos destacado la productividad que han tenido las hipótesis históricas más importantes del siglo XIX y principios del XX - por ejemplo, las teorías Evolutiva, Orbital y de Deriva Continental ${ }^{6}$-, rendimiento que pone en tela de juicio la validez de un método uniforme en la práctica de las ciencias naturales.

\section{SingulARIDADES DE LA BIOLOGÍA COMO HISTORIA NATURAL}

Las preguntas que interesan al naturalista abordan la historia, origen y evolución de los organismos vivos, todas cuestiones que conllevan una mirada distinta a la que impera en otros ámbitos de la biología y de las ciencias naturales históricas en general. Fuentes (1983) sostiene que existen dos concepciones distintas en biología: a) por una parte, la concepción "fisiológica" - preocupada de los sistemas desde el punto de vista de su funcionamiento $\mathrm{y}$ en donde su historia es irrelevante- $\mathrm{y}$, por otra, b) la concepción "naturalista", que considera la historia de los organismos en el tiempo y el espacio. Fuentes y Sánchez (1982) y Fuentes (1983) distinguen tres clases de preguntas de la investigación naturalista: a) preguntas acerca de la distribución de los organismos en el espacio y tiempo (ámbito de la biogeografía), b) preguntas que se refieren al ámbito de distribución de los caracteres de distintos grupos de organismos (ámbito de la morfología y la sistemática), y c) preguntas relativas a la interconexión entre los espacios fenotipos/tiempo y biósfera/tiempo de los organismos (ámbito de la variación ontogenética y filogenética). Según estos autores, "algunos de los eventos que subyacen la distribución y abundancia de organismos en el dominio biósfera/tiempo pueden traducirse en cambios de ubicación relativa en el ámbito fenotipos/tiempo" (Ibíd.). Todas estas clases de preguntas propias de la Historia Natural tienen en común la búsqueda del "por qué" de la distribución en los distintos ámbitos señalados, en contraste con la perspectiva del "fisiólogo", a quien le interesa el "cómo", es decir, la función específica de una estructura, de un organismo, o incluso de una distribución. Como señalamos previamente, a

${ }^{6}$ En la actualidad estas hipótesis históricas son comunes en geología, paleontología, arqueología, biología evolutiva, astronomía y astrofísica. 
partir de Darwin la conformidad histórica de los seres vivos se comprende a través de la relación ancestro-descendencia.

La singularidad histórica de los sistemas biológicos exige la comprensión de ciertos principios exclusivos de los seres vivos, además de los compartidos con las ciencias naturales históricas en general y que hemos revisado en la primera parte de este trabajo. Antes de referirnos a algunos ejemplos de conceptos y metodologías claves en la investigación de los seres vivos, consideramos que hay que destacar dos principios que nos parecen fundamentales: conformidad a fin del organismo y acoplamiento estructural organismo-espacio de vida.

\subsection{ORGANISMO Y CONFORMIDAD INTERNA}

En 1790, en la Crítica del Juicio (CJ), Kant introdujo por primera vez la doctrina del "organismo", para referirse a la "conformidad intrínseca" (innere Zweckmassigkeit) que exhiben los "seres organizados" -nombre que se daba en la época a los seres vivos ${ }^{7}$. Este autor introdujo la idea de conformidad interna o intrínseca del organismo como concepto reflexivo, con el propósito de hacer comprensible la peculiaridad del dominio de la vida al discernimiento humano. Expresa así lo que se entiende por conformidad intrínseca: "Para que una cosa que es una producción de la naturaleza, al propio tiempo sea reconocida únicamente como un fin de la misma, es necesario que contenga una relación recíproca de causa y efecto" (CJ, § 65 p. 232). En otras palabras, las partes concurren entre sí y con el todo, de manera que son, alternativamente, causa y efecto del conjunto integrado. Así, este principio de conformidad atribuye a los seres vivos una nueva causalidad, además de la causalidad eficiente, noción heurística válida solamente para la reflexión sobre la vida. Según Kant: "producto organizado de la naturaleza es aquel en que todo es fin y también medio. Nada en él es porque sí, sin fin o atribuible a un mecanismo natural ciego" (CJ, § 66 p. 236). Con la doctrina del organismo Kant introduce por primera vez en las ciencias naturales la idea de autoorganización, -que da cuenta de las propiedades de autodeterminación, automantención y autoreproducción- $\mathrm{y}$ fundamenta la singularidad del objeto de estudio de la biología ${ }^{8}$. En 1972 Maturana acuña el término autopoiesis -del griego poiesis, creación, producción; auto, por sí mismo- para referirse a la autoorganización que caracteriza los sistemas vivos (Maturana \& Varela 1979)9. "Autopoiesis" significa una dinámica de cambio o flujo organizador continuo que involucra constitutivamente una dinámica histórica. El valor

${ }^{7}$ La palabra "seres o entes organizados" (organized beings) es introducida por Buffon, contemporáneo de Kant, para designar a los seres vivos.

${ }^{8} \mathrm{La}$ palabra biología es usada desde finales del siglo XVIII por Roose y principios del XIX por Burdach y Lamarck.

${ }^{9}$ Contrapone el término autopoiesis a heteropoiesis, que designa al espacio del diseño humano. heurístico del concepto de auto-organización en las ciencias naturales ha sido destacado por Keller (2008).

\subsection{Mundo Circundante: Acoplamiento estructural} ORGANISMO-ÁMBITO DE VIDA

Una versión previa del principio de acoplamiento estructural procede de la etología y fisiología animal y fue introducido por Uexküll (1913), quien, siguiendo a Kant, entiende la conducta animal como una conformidad extrínseca. El propone el concepto de "mundo circundante" (Umwelt) o "mundo perceptivo" (Merkwelt) para designar la unidad que conforma el organismo con el conjunto de señales o "notas" del entorno que percibe y ante el que reacciona. Uexküll llama "conducta" a la capacidad del organismo de circunscribir este espacio propio que contiene los desinhibidores de sus impulsos y con los cuales conforma una unidad que él llama círculo funcional. Esta concepción de la conducta animal confiere relevancia a los cambios que experimentan tanto el organismo como su entorno acoplado, en otras palabras, co-evolución de ambos a través de una "historia"10 común. Bateson (1979) vincula los conceptos de conformidad interna y externa con la idea central de la teoría evolutiva, la adaptación ${ }^{11}$, el proceso de ajuste del organismo a la porción de entorno que le es propia. Maturana y Mpodozis (2000) acuñan el término "acoplamiento estructural" en el marco de una nueva teoría evolutiva en la que el ensamble organismo-condición de vida queda determinado en un contexto histórico, y constituye el eje central del devenir de los organismos. El valor heurístico del concepto en ecología evolutiva y biogeografía histórica es incuestionable.

\subsection{Algunas metodologías históricas propias de LA} BIOLOGÍA

Los dos principios comentados permiten establecer un límite entre la materia no organizada y los "organismos", como asimismo entre estos últimos y cualquier diseño tecnológico producido por el hombre. Por otro lado, la especial consideración histórica de la mirada del naturalista vincula los aportes de una serie de disciplinas que muchas veces son consideradas erróneamente como separadas, en ocasiones como obsoletas: morfología comparada, paleontología, biogeografía, embriología y, prioritariamente, sistemática botánica y zoológica. Ellas aportan los elementos centrales para la formulación de hipótesis históricas en biología. Como no es el propósito de este trabajo analizar las metodologías particulares de cada disciplina que constituye el núcleo central del dominio de

\footnotetext{
${ }^{10}$ Bateson G. (1979, p. 12-13) llama "conducta" a "esas historias proyectadas en la acción".

${ }^{11}$ Bateson (1979, p. 195): "Hay dos modos de lograr la acción adaptativa. La evolución debe siempre (como las caras de Jano) mirar en dos direcciones: hacia adentro, en dirección a las regularidades del desarrollo y la fisiología del ser vivo, y hacia afuera, en dirección a las caprichosas exigencias del ambiente".
} 
la Historia Natural de los organismos, queremos solamente destacar algunos conceptos que se desprenden de los principios mencionados y que son comunes a la indagación metódica de todas estas disciplinas. Este es el caso, por ejemplo, de los conocidos conceptos de homología y analogía, los cuales adquieren un sentido como producto de una historia genealógica/filogenética y resultaron de los estudios del plan de organización común y similitudes morfológicas establecidas por los primeros naturalistas. A continuación nos interesa destacar con algo más de detalle el concepto de anomalía.

\subsubsection{ANOMALÍAS MORFOLÓGICAS}

La investigación metódica de anomalías es atribuida a Darwin y llamada por Gould (1980 ) "Principio Panda", cuando se refiere al uso de órganos vestigiales -como el famoso pulgar del oso panda-. El concepto es usado por paleontólogos, morfólogos y también biogeógrafos. El fundamento de su uso reside en el hecho que las irregularidades de estructuras y/o formas de organismos, o distribuciones geográficas de comunidades o biotas, permiten postdicciones y planteamiento de hipótesis sobre acontecimientos históricos pasados.

De acuerdo a Gould (1980), los detallados y aparentemente inútiles estudios anatómicos de Darwin en orquídeas y en animales son ejemplos de una búsqueda de imperfecciones y rarezas en la naturaleza, en franca oposición a la búsqueda de sofisticados diseños que sugiere a muchos pensadores creacionistas la idea de un diseñador inteligente. El caso del "pulgar" del panda es un buen ejemplo de esto. El panda, a diferencia de sus parientes más cercanos omnívoros, se alimenta exclusivamente de bambú, para lo cual se sirve de una extraña adaptación de sus patas delanteras: además de sus cinco dedos, posee una estructura especial que asemeja a un sexto dedo oponible -el así llamado "pulgar del panda"-. Esta estructura corresponde a un pequeño hueso de la muñeca que se ha alargado hasta igualar la longitud de los huesos de los dedos, con una concomitante reconfiguración de la musculatura asociada, estructura separada de los otros dígitos por un surco entre las almohadillas que sirve como canal para deshojar las varas de bambú. Un ejemplo botánico usado por Darwin es el de las variadas configuraciones que adquiere el pétalo inferior de todas las orquídeas -el labelo- que conduce al insecto polinizador hacia el ginnostemio que se encuentra en su base (gineceo y androceo fusionados). Darwin describe este despliegue de formas como "a prodigality of resources for gaining the very same end, namely, the fertilization of one flower by pollen from another plant". Precisamente estos ejemplos de formas bizarras generadas a partir del reacomodo de materiales preexistentes apoyan la idea de Darwin, en contraposición a la idea dominante de diseños preestablecidos intencionalmente.

\subsubsection{Anomalías biogeográficas: Disyunciones}

Las anomalías en la distribución geográfica de especies, que Darwin nota durante el viaje del Beagle por la costa de Sudamérica, constituyen uno de los fundamentos más importantes de su teoría, como lo dice en la introducción del Origen de las Especies. Particularmente, la observación de que poblaciones de especies emparentadas varían en sus características cuando se encuentran aisladas, como por ejemplo los pinzones de las islas Galápagos; o de distribuciones aisladas de biotas similares en cimas de cerros, explicadas por Darwin (1859) sobre la base de desplazamientos espaciales de organismos durante las eras glaciales. Esta explicación es notable si se considera que los fenómenos glaciales eran apenas conocidos en su época.

A principios del siglo XX la investigación geológica experimentó un notable avance con la teoría de deriva continental planteada por Alfred Wegener en 1915, y reformulada en 1962 sobre sólidas bases empíricas como teoría de tectónica de placas. Paralelamente, la teoría orbital de Milankovich aportó las bases para entender la variación climática en períodos mileniales. Así, la historia filogenética de los organismos y del escenario físico asociado convergen en una síntesis a fines del siglo XX, facilitada por el establecimiento de filogenias usando herramientas moleculares para definir homologías. Recién entonces la Historia Natural de los seres vivos puede ser concebida como un dominio unificado sobre la base de principios e hipótesis históricas. La incorporación de este entramado teórico permite interpretar las anomalías distribucionales de organismos y comunidades -o disyunciones geográficas con valor histórico- como la expresión de procesos biológicos acoplados a grandes cambios geológico-climáticos que no tienen correlato en el presente.

\section{CONCLUSIÓN}

Como conclusión, nos parece interesante resaltar nuestra contribución al debate actual acerca de la validez y diversidad de metodologías en el quehacer científico. Como lo han destacado otros autores, opinamos que la creencia en un sólo método científico, de práctica uniforme y transdisciplinario, es infundada (Feyerabend 1975, Armesto 1985, Willson \& Armesto 2006, Cleland 2001, 2002, 2011). Considerando el cuestionamiento que se hace a menudo al estatus científico de la investigación naturalista, el énfasis de nuestra argumentación ha sido precisar los principios y metodologías que definen el campo de las ciencias naturales históricas, especialmente el de la Historia Natural. Esta definición adquiere especial relevancia si consideramos el incipiente desarrollo de las ciencias naturales históricas en nuestro país y el creciente interés en la biogeografía histórica, paleoecología y reconstrucción climático- 
vegetacional en nuestro territorio, incluyendo herramientas como la filogeografía y la modelación de nichos.

Las ideas discutidas abren posibilidades de reflexión en torno a la interfase biología-filosofía. Aunque este tema es aún rudimentario a nivel nacional e internacional, destacan esfuerzos recientes en este ámbito, por ejemplo el aporte de Rozzi (2012), quien analiza la biodiversidad, política y ética ambiental. Nuestro texto inicia el análisis de algunas ideas en esta interfase, en particular el valor heurístico de principios centrales en torno a la historia natural, como son los principios de conformidad afin y de acoplamiento estructural de los organismos vivos.

\section{LITERATURA CITADA}

Alvarez, L.W., W. Alvarez, F. Asaro \& H.V. Michel. 1980. Extraterrestrial Cause for the Cretaceous Tertiary Extinction. Science 208: 1095-1108.

Ariew, A. 2007. Teleology. In D. Hull \& M. Ruse (eds.), Cambridge Companion to Philosophy of Biology, pp. 160181. Cambridge. Cambridge University Press.

Armesto, J.J. 1985. Prueba de hipótesis en ecología: Una crítica al método científico formal. Revista Chilena de Historia Natural 58: 107-108.

Bateson, G. 1979. Mind and Nature: A Necessary Unity. New York, Bantam Books. 238 pp.

Cleland, C.E. 2001. Historical science, experimental science, and the scientific method. Geology 29: 987-990.

Cleland, C.E. 2002. Methodological and Epistemic Differences between Historical Science and Experimental Science. Philosophy of Science 69: 474-496.

Cleland, C.E. 2011. Prediction and Explanation in Historical Natural Science. The British Journal for the Philosophy of Science 62: 1-32.

Darwin, C. 1859. The Origin of Species by Means of Natural Selection. First Edition, London: Murray, Reedited by E Mayr, 1964, Cambridge MA: Harvard University Press. $486 \mathrm{pp}$.

Feyerabend, P. 1975. Contra el Método. Traducción español de 1993. Barcelona: Planeta De-Agostini S. A. 189 pp.

Fuentes, E.R. 1983. Pensamiento fisiológico y evolutivo en el quehacer de los naturalistas. Revista Chilena de Historia
Natural 56: 89-90.

Fuentes, E.R. \& P. SÁnchez. 1982. ¿Qué hacen los naturalistas? Carta abierta a Luco. Archivos de Biología y Medicina Experimentales 15: 491-499.

Gould, S.J. 1965. Is Uniformitarianism necessary? American Journal of Science 263: 223-228.

Gould, S.J. 1980. The Panda's Thumb: More reflections in Natural History. New York, W. W. Norton \& Co. 352 pp.

Gould, S.J. 1986. Evolution and the Triumph of Homology, or Why History Matters. American Scientist 74: 60-69.

JAKSIC, F. 1999. ¿Qué fue de la Historia Natural? Revista Chilena de Historia Natural 72: 5-6.

Kant, E. 1790. Crítica del juicio. Traducción José Rovira Armengol. Buenos Aires: Editorial Losada, 2005. 365 pp.

Keller, E.F. 2008. Organisms, Machines, and Thunderstorms: A History of Self-Organization, Part One. Historical Studies in the Natural Sciences 38: 45-75.

Lyell, S.C. 1989. Principles of Geology (Vol. 1). Bishen Singh Mahendra Pal Singh. 511 pp.

Maturana, H. \& F.J. Varela. 1979. Autopoiesis and Cognition. The Realization of the Living. Boston Studies in the Philosophy of Science 42: 1-141.

Maturana, H. \& J. Mpodozis. 2000. The origin of species by means of natural drift. Revista Chilena de Historia Natural 73: 261-310.

Peters, R.H. 1980. Useful concepts for predictive ecology. Synthese 43: 257-259.

Rozzi, R. 2012. South American Environmental Philosophy: Ancestral Amerindian Roots and Emergent Academic Branches. Environmental Ethics 34:4: 343-366.

Rozzi, R., E. Hargrove, J.J. Armesto, S. Pickett \& J.J. Silander. 1998. "Natural drift" as a Post-Modern evolutionary metaphor. Revista Chilena de Historia Natural 71: 5-17.

Sober, E. 2001. Venetian Sea Levels, British Bread Prices, and the Principle of the Common Cause. The British Journal for the Philosophy of Science 52: 331-346.

Turner, D. 2004. Local Underdetermination in Historical Science. Philosophy of Science, 72: 209-230.

UeXKÜll, J. von. 1913. Ideas para una concepción biológica del mundo. Trad. R.M. Tenreiro. $2^{\text {a }}$ ed. 1945. Buenos Aires: Espasa-Calpe. 244 pp.

Willson, M. \& J.J. Armesto. 2006. Is natural history really dead? Toward the rebirth of natural history. Revista Chilena de Historia Natural 79: 279-283.

Recibido: 28.01.14

Aceptado: 17.04 .14 